Studiengemeinschaft verdient auch er ein großes Dankeschön.

Touristische Höhepunkte des Jubiläumstreffens waren Ausflüge in das Naturschutzgebiet Georgenfelder Hochmoor entlang der Grenze zur Tschechischen Republik und eine Liftfahrt vom Mückenberg (Komari hurka) nach Grauben (Krupka) mit Besichtigung der Klosteranlagen Mariaschein.

Einen würdigen Abschluss am letzten Tag unseres Treffens bildete ein Besuch des Osterzgebirgsmuseums im Renaissanceschloss Lauenstein. Für uns besonders interessante Schwerpunkte der Ausstellung waren u. a. die Geschichte der Kursächsischen Postmeilensäulen, das Leben und Wirken des Baumeisters der Dresdner Frauenkirche, George Bähr, und beeindruckende Landschaftsmodelle des Osterzgebirges.

Die Unterzeichner dieser Zeilen gehen davon aus, dass es auch im Jahr 2015 wieder ein Treffen der ehemaligen "K1" geben sollte und auch geben wird.

Kurt Ziesing, Erfurt und

Rolf Dörrstock, Bonn

\section{Langjährige}

DGfK-Mitgliedschaft

Juli / August 2015

Folgenden Mitgliedern dankt die Deutsche Gesellschaft für Kartographie für ihre langjährige Treue:

- Mitglied seit 60 Jahren: Dipl.-Ing. Hans-Oswald Kessler, 70378 Stuttgart

mitglied seit 50 Jahren: Dipl.-Ing. Hans-Joachim Paul, 53424 Remagen

\section{Mitglied seit 25 Jahren:}

Dipl.-Ing. Bernd Rappe,

30966 Hemmingen-Westerfeld Dipl.-Ing. (FH) Susanne Friedeheim, 52074 Aachen

Dipl.-Geogr. Ingo Wolff,

44135 Dortmund

Dipl.-Ing. (FH) Anja Hopfstock, 63579 Freigericht-Altenmittlau Dipl.-Ing. (FH) Stephan Pohl, 30453 Hannover

Dipl.-Ing. (FH) Barbara Böttcher-

Walkowiak, 13465 Berlin

Michael Recke, 26725 Emden

\title{
Veranstaltungskalender 2015
}

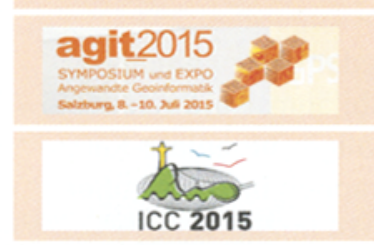

8. bis 10. Juli 2015, Salzburg, Österreich AGIT 2015 - Symposium und Expo für Angewandte Geoinformatik Universität Salzburg, Department for Geoinformatics. http://www.agit.at

23. bis 28. August 2015, Rio de Janeiro, Brasilien $27^{\text {th }}$ International Cartographic Conference International Cartographic Association (ICA). http://www.icc2015.org/

INTER G E $\mathbf{O}^{\circ}$ (I)

15. bis 17. September 2015, Stuttgart Intergeo und 63. Deutscher Kartographentag

DVW Gesellschaft für Geodäsie, Geoinformation und Landmanagement e. V., Deutsche Gesellschaft für Kartographie http://www.de.roombites.com/intergeo-2015_-deutschland

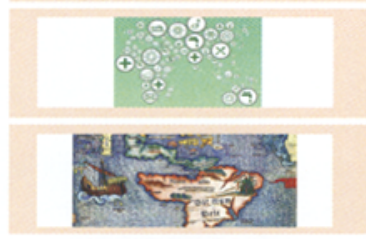

\section{AgA Tagung}

16. bis 18. September 2015, Augsburg LBS2015 - $12^{\text {th }}$ conference on Location-Based Services www.lbs2015.tum.de

13. September bis 6 . Dezember 2015, Lemgo Weltvermesser - Das Goldene Zeitalter der Kartographie Weserrenaissance-Museum Schloß Brake. www.weltvermesser.de

21. September bis 22. September 2015, Erfurt 52. Aga-Tagung

Landesamt für Vermessung und Geoinformation Thüringen http://www.ikg.uni-hannover.de/aga/index.php?id=343

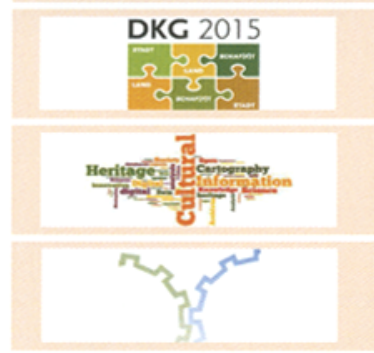

1. bis 6. Oktober 2015, Berlin

DKG 2015 - Deutscher Kongress für Geographie DGfG. http://www.dkg2015.hu-berlin.de

28. bis 30. Oktober 2015, Berlin Digital Cultural Heritage CODATA-Germany, DGfK, ICA u.a. http://dch2015.net/

3. und 4. November 2015, Bonn

Workshop "3-D-Stadtmodelle"

Gemeinsame DGfK/DGPF-Kommission 3-D-Stadtmodelle. www.3d-stadtmodelle.org

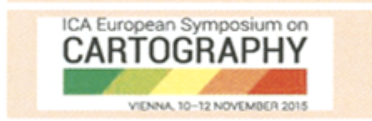

10. bis 12. November 2015, Wien

$1^{\text {st }}$ ICA European Symposium on Cartography

TU Wien, Institut für Kartographie - Deadline abstract: 01.06.2015 http://eurocarto.org

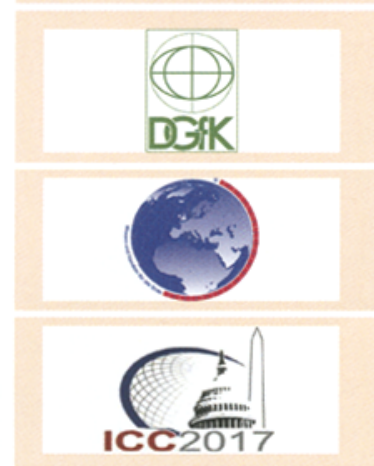

2016

14. bis 16. Juni 2016, Potsdam

64. Deutscher Kartographentag

Deutsche Gesellschaft für Kartographie. Internetadresse demnächst

11. bis 13. Oktober 2016, Hamburg

Intergeo 2016

DVW Gesellschaft für Geodäsie, Geoinformation und Landmanagement e. V. Internetadresse demnächst

2017

2. bis 7. Juli 2017, Washington, USA

$\mathbf{2 8}^{\text {th }}$ International Cartographic Conference

International Cartographic Association (ICA). http://www.icc2017.org/

\section{Geburtstage}

Juli / August 2015

1.7. Dipl.-Ing. Heiko Taubenrauch, 49661 Cloppenburg, 60 Jahre

6.7. Dr. Helga Kallenbach,

14109 Berlin, 80 Jahre

11.7. Prof. Dr.-Ing. Peter Mesenburg, 45147 Essen, 75 Jahre

12.7. Professor Uwe Ulrich Jäschke, 01109 Dresden, 60 Jahre

16.7. Prof. Dr.-Ing. Rüdiger Finsterwalder, 82131 Gauting, 85 Jahre
23.7. Fritz Heubner, 59505 Bad Sassendorf, 91 Jahre 28.7. Edeltraud Mohaupt-Wittrup, 48167 Münster, 65 Jahre

8.8. Dr. Annelie Mehlhorn, 09117 Chemnitz, 65 Jahre 14.8. Dipl.-Ing. (FH) Bernhard Horst, 30659 Hannover, 80 Jahre

19.8. Dipl.-Ing. Heidemarie Müller, 01309 Dresden, 65 Jahre

19.8. Joachim Pestke,

13465 Berlin, 60 Jahre
21.8. Dipl.-Ing. (FH) Gerd Heit, 49078 Osnabrück, 65 Jahre 24.8. Dipl.-Ing. (FH) Manfred Starke, 45136 Essen, 75 Jahre

25.8. Dipl.-Ing. (FH) Helmut Lehmann, 76307 Karlsbad, 80 Jahre

25.8. Mag. phil. Kasimir Szarawara, 8010 Graz, 60 Jahre

26.8. Dipl.-Ing. Kurt Ziesing, 99089 Erfurt, 80 Jahre 29.8. Hans-Ulrich Wetterhahn, 60435 Frankfurt am Main, 65 Jahre 